\title{
Clinical Significance, Antibiotic Resistance and Biofilm Formation of
} Methicillin Resistant Staphylococcus aureus: Review

\author{
Sindhu Sasidhar ${ }^{*}$ \\ Department of Biotechnology, Karunya Institute of Technology and Sciences, India
}

"Corresponding author: Sasidhar S, Department of Biotechnology, Karunya Institute of Technology and Sciences, India, Tel: +91 4222614300; E-mail: sasidharsindhu@gmail.com

Received: August 15, 2018; Accepted: August 24, 2018; Published: August 27, 2018

\begin{abstract}
Staphylococcus aureus is an emerging crisis in medical field due to its development of antibiotic resistance. It is capable of causing numerous hazardous infections and continued medication has led to the development of antibiotic resistance. Moreover, $S$. aureus is capable of biofilm formation which is a major cause of multidrug resistance. It causes a wide range of infections starting from mild infections to life threatening diseases. $S$. aureus is considered as a major threat to living population. It has been reported that day by day the strain is becoming resistant to the conventional treatments, so new or alternative medication or treatment is an emergency. In this review the clinical significance, antibiotic resistance and the biofilm formation of $S$. aureus has been discussed.
\end{abstract}

Keywords: S. aureus; Clinical significance; Biofilm formation; Antibiotic resistance

\section{Introduction}

\subsection{Methicillin resistant Staphylococcus aureus and its clinical significance}

Staphylococcus aureus appears in cocci shape and is gram-positive, which appears in grape-like clusters. Methicillin Resistant $S$. aureus is any strain of $S$. aureus that has developed through the process of natural selection, resistant to a wide range of $\beta$-lactam antibiotics [1]. S. aureus was demonstrated to be a major human pathogen capable of causing a wide range of infections, from relatively mild skin infections such as folliculitis and furunculosis to life-threatening conditions, including sepsis, deep abscesses, pneumonia, osteomyelitis, and infective endocarditis [2]. In 2013, the Centers for Disease Control and Prevention (CDC) reported that more than 11,000 people died from a methicillin-resistant S. aureus (MRSA)-related infection in the United States [3]. It is found mostly under the anterior nostrils, respiratory tract, open wounds, intravenous catheters and urinary tract are also potential sites of infection [4]. Some healthy individuals may carry MRSA without any symptoms for periods ranging from few weeks to many years. Comprised immune system patients are at higher risks of secondary infection which is symptomatic [5].

Citation: Sasidhar S. Clinical Significance, Antibiotic Resistance and Biofilm Formation of Methicillin Resistant Staphylococcus aureus: Review. Clin Case Rep Open Access. 2018;1(1):102. 
www.yumedtext.com | August-2018

MRSA is classified into two major classes healthcare-associated (HA-MRSA) as well as community-associated (CA-MRSA). Both are capable of causing a wide range of diseases, including endocarditis, pneumonia, osteomyelitis, toxic-shock syndrome, carbuncles and food poisoning [2]. In early 1940s, mortality among 122 consecutive patients were 82\% and in those aged above 50 years were $98 \%$. In modern era, it is estimated that $25 \%-30 \%$ of healthy individuals carry S. aureus on the skin or mucous membranes [6,7]. S. aureus has always been a stumbling block for antimicrobial chemotherapy, and have overcome all the therapeutic agents that have been developed in past 50 years. The most appropriate example is methicillin resistant $S$. aureus, the first clinical isolate which was reported in early 60s, just one year after the launch of methicillin. Since then, MRSA has gradually disseminated, and in the 1970s began causing serious hospital infection worldwide [8-11]. It is found that in India, the population of MRSA is greater in hospital population than in the community, which is the major cause of hospital-acquired wound infections due to accidental injury or surgery and therefore these strains generally show multiple drug resistance, which limits treatment possibilities [12].

\subsection{Antibiotic resistance of bacteria}

The overuse, misuse and lack of new antibiotics have led to the development of drug resistant bacteria which is a public menace [8]. These bacterial infections with multiple drug resistance may increase the mortality and morbidity in Intensive Care Unit patients [9]. The multidrug resistance of bacteria occurs mainly due to two mechanisms: a) accumulation of multiple genes on resistance $(\mathrm{R})$ plasmids, with each coding for resistance to a single drug, within a single cell; b) by the action of multidrug efflux pumps, each of which can pump out more than one drug type [10]. Examples of few drug resistant microbes include: i) E. coli resistant to cephalosporins and fluoroquinolones, ii) Klebsiella pneumoniae resistant to cephalosporins and carbapenems, iii) S. aureus resistant to methicillin, iv) Streptococcus pneumoniae resistant to penicillin, v) Non-typhoidal Salmonella resist to fluoroquinolones, vi) Shigella species resistant to fluoroquinolones, vii) Neisseria gonorrhoeae resistant to cephalosporins, viii) Mycobacterium tuberculosis resistant to rifampicin, isoniazid and fluoroquinolones [7,11].

\subsection{Antibiotic resistance of $S$. aureus}

S. aureus acquires resistance by mutation, conjugation, transduction or transformation. The resistance due to transformation and transduction are rare in case of staphylococci. The chromosomal mutation generally causes an altered antimicrobial target of action; it also affects the bacterial membrane permeability to antimicrobials or inactivates it enzymatically. The alteration of $\beta$-lactam target site may also cause resistance and this is the major mechanism involved in methicillin resistance in $S$. aureus [7,6]. Barakat et al. [13] reported that mupirocin an antibiotic which was playing a crucial role in eradication of MRSA by inhibiting the protein synthesis of the strain and which was used in the treatment of skin infections started showing low and high level resistance due to the continuous increased usage of the drug. It has been reported that the organism acquires resistance as soon as the entrance of new antibiotics, for example $S$. aureus resistance to penicillin was reported within 4 years of entrance of penicillin G into the market. The mutation caused in DNA gyrase (gyr) and topoisomerase IV $(\mathrm{grl})$ gene, results in the resistance of quinolone antibiotics, while the mutation in RNA polymerase gene ( $r p o B$ ) indicates the resistance to rifampicin [5].The development of resistance may be due to horizontal gene transfer or else mutations in the genes of chromosomes [14]. 
www.yumedtext.com | August-2018

The resistance of $S$. aureus to $\beta$-lactams is due to the addition of non-native gene which encodes penicillin-binding protein PBP2a. The enzyme $\beta$ lactamase also known as penicillinase inactivates the drug by the hydrolization of the $\beta$ lactam ring. It has been found that more than $90 \%$ of $S$. aureus showed resistance to penicillin. The term methicillin resistant $\beta$ lactams indicates resistance to all $\beta$ lactam antibiotics except the new generation cephalosporin $\beta$ lactams. It has been reported that vancomycin is the considered as the last treatment along with new formulations like linezolid and daptomycin for severe infections of MRSA [15]. Daptomycin which is a lipopeptide antibiotic, usually used in the treatment of various infections caused by gram-positive bacteria. Recently, it has been reported that the patients undergoing DAP therapy are developing resistance, it is due to the point mutations in several genes [16]. The infections caused by MRSA are usually treated with Trimethoprim-sulfamethoxazole and clindamycin; recently it was reported to develop resistance. The resistance increased from $9.3 \%$ in 2005 to $16.7 \%$ in 2014 which was reported by a study conducted in Military Health system in US. Moreover, the MRSA started showing resistance to mupirocin and chlorhexidine. The plasmid mediated mupA gene encoding RNA synthetase is a primary factor for the development of mupirocin resistance. The gene responsible for chlorhexidine resistance $q a c A / B$ encodes efflux pumps and are also plasmid mediated [17]. It is reported that the multidrug resistance infections has raised from $2.5 \%$ to $8.6 \%$ over last twenty years [18].

\section{Bacterial Biofilms}

Biofilm is a group of microbial cells that is permanently associated with a surface and surrounded by matrix, chiefly composed of polysaccharide material. Biofilms are difficult to eradicate [19]. It is nothing but communities of microorganisms within extracellular polymeric matrix which comprises of lipids, proteins, polysaccharides and nucleic acids, they attach to a biotic or abiotic surface in the solid-liquid interface. Moreover the organisms in the biofilm are provided with essential nutrients supply and water for their growth [20]. The biofilm formation is the major cause of many chronic infections. Moreover they are multidrug-resistant and cause failure of the treatment. The eradication of biofilm is not possible using conventional antibiotics [21]. According to Elizabeth [22] biofilm formation is a resistant mechanism utilized by bacteria against immune system or antibiotics. They also reported that compared to the biofilm formed by mono-species organisms the biofilm formed by multi-species organisms is structurally and chemically more complex. Moreover, the adhesion forces vary between mono-species and multi-species biofilms. The biofilm formation depends on certain factors like a) availability of key nutrients, b) motility of bacteria, c) chemotaxis towards surface, d) surface adhesion and e) presence of surfactants. Biofilms are the site of quorum sensing [23].

\subsection{Biofilm formation of S. aureus}

Biofilm formation is the vital reason for antibiotic resistance and is one of the major virulence factors of MRSA. The formation of biofilm leads to recurrent infections especially in burn patients. Biofilms are capable of attaching to living as well as inanimate objects; this attachment to the surfaces is considered as the first stage of infection. The attachment of organisms to each other is caused by polysaccharide intracellular adhesion (PIA) leading to biofilm formation. Polysaccharide intracellular adhesion (PIA) is a synthesized by-product of icaABCD operon [23]. Therefore, the capability of S. aureus in formation of biofilm on surface of medical devices is a key factor contributing to nosocomial infections. In catheterized patients the formation of biofilm is carried out in two steps: i) the bacterium attaches to the inert surfaces and starts growing, ii) Multilayer formation due to multiplication and proliferation of bacteria [24]. It is found that the structure of biofilm ranges from monolayer to multilayer with macroscopic dimensions. Therefore, the chemical composition is very 
www.yumedtext.com | August-2018

much dynamic in biofilm, and it promotes the exchange of nutrients. Moreover, they have the capability of sharing the genetic material between the communities. The polymer production is the major key weapon used by biofilm to promote themselves to a region of nutrient and oxygen [25].

In diary industry, the contamination of dairy products is mainly due to the bacterial biofilms. It has been found that poor GMP (Good Manufacturing Practices) in dairy processing leads to the spread of S. aureus and further leads to biofilm formation [26]. The biofilms are highly tolerant against antibiotic treatment and they differ physiologically in planktonic state. The biofilm formation differs in methicillin-sensitive $S$. aureus as well as methicillin-resistant $S$. aureus by the encoding gene producing polysaccharide intercellular adhesion protein. In MSSA, icaADBC gene is responsible for the production of PIA, whereas in MRSA the biofilm formation is independent of ica. Moreover, the biofilm formed by MRSA has serious clinical impacts [27].

\section{Conclusion}

S. aureus, one of the most deadly nosocomial pathogen that is found all around the globe. The emergence of S. aureus and its biofilm production with resistance to many groups of antibiotics is a growing concern worldwide. The irrational use of antibiotics against this fatal pathogen has led to increased resistance due to which an alternate effective therapy is an important requirement. Therefore, regular monitoring is required to find out a novel alternative. There is a great challenge for the clinician to find out ways to combat ever increasing multidrug resistance.

\section{REFERENCES}

1. Mirani ZA, Aziz M, Khan MN, et al. Biofilm formation and dispersal of Staphylococcus aureus under the influence of oxacillin. Microb Pathog. 2013;61:66-72.

2. Rahimi G, Alizadeh F, Khodavandi A. Mycosynthesis of Silver Nanoparticles from Candida albicans and its Antibacterial Activity against Escherichia coli and Staphylococcus aureus. Trop J Pharm Res. 2016;15(2):371-5.

3. Thangamani S, Younis W, Seleem MN. Repurposing celecoxib as a topical antimicrobial agent. Front Microbiol. 2015;6:750.

4. Yan M, Pamp SJ, Fukuyama J, et al. Nasal microenvironments and interspecific interactions influence nasal microbiota complexity and S. aureus carriage. Cell Host Microbe. 2013;14(6):631-40.

5. Muhlebach S, Christopher H (2011) Review: Staphylococcus aureus and MRSA in cystic fibrosis. J Cyst Fibros. 2011;10(5):298-306.

6. Cuny C, Witte W. MRSA in equine hospitals and its significance for infections in humans. Vet Microbiol. 2016;200:59-64.

7. Rajamuthiah R, Fuchs BB, Conery AL, et al. Repurposing salicylanili anthelmintic drugs to combat drug resistant Staphylococcus aureus. PLoS One. 2015;10(4):e0124595.

8. Lau QY, Tan YF, Goh VC, et al. An FDA-drug library screen for compound with bioactivities against meticillin-resistant Staphylococcus aureus (MRSA). Antibiotics (Basel). 2015;4(4):424-34.

9. Maviglia R, Nestorini R, Pennisi M. Role of old antibiotics in multidrug resistant bacterial infections. Curr Drug Targets. 2009;10(9):895-905.

10. Nikaido H. Multidrug Resistance in Bacteria. Annu Rev Biochem. 2009;78:119-46. 
www.yumedtext.com | August-2018

11. Tanwar J, Das S, Fatima Z, et al. Multidrug resistance: an emerging crisis. Interdiscip Perspect Infect Dis. 2014;7:110-9.

12. Rump B, De Boar M, Reis R, et al. Signs of stigma and poor mental health among carriers of MRSA. J Hosp Infect. 2016;95(3):268-74.

13. Barakat GI, Nabil YM. Correlation of mupirocin resistance with biofilm production in methicillin-resistant Staphylococcus aureus from surgical site infections in a tertiary centre, Egypt. J Glob Antimicrob Resist. 2017;4:1620.

14. Timothy FJ. Antibiotic resistance in Staphylococcus aureus current status and future prospects. FEMS Microbiol Rev. 2017;41(3):430-49.

15. Sharon PJ, Gavin PK. Mechanisms of Methicillin Resistance in Staphylococcus aureus. Ann Rev Biochem. 2015;84:577-601.

16. Muller A, Grein F, Otto A, et al. Differential daptomycin resistance development in Staphyloccocus aureus strains with active and mutated gra regulatory systems. Int J Med Microbiol. 2017;308(3):335-48.

17. Rachel ML, Latania LK. The Growing Threat of Antibiotic Resistance in Children. Infect Dis Clin North Am. 2018;32(1):1-17.

18. Sheridan R, Weber J, Chang $\mathrm{P}$, et al. Multi-drug resistant gram negative bacteria colonization and infection in burned children: Lessons learned from a 20-year experience. Burns Open. 2018;2(1): 43-6.

19. Shineman DW, Alam J, Anderson M, et al. Overcoming obstacles to repurposing for neurodegenerative disease. Ann Clin Transl Neurol. 2014;1(7):512-8.

20. Alvarado-Gome E, Perez-Diaz M, Valdez-Perez D, et al. Adhesion forces of biofilms developed in vitro from clinical strains of skin wounds. Mater Sci Eng. 2017;82:336-44.

21. Banu A, Hassan MMN, Rajkumar J, et al. Spectrum of bacteria associated with diabetic foot ulcer and biofilm formation: A prospective study. Australas Med J. 2015;8(9):280-5.

22. Elizabeth AS, Townsley L. Natural-Product Antibiotics: Cues for Modulating Bacterial Biofilm Formation. Trends Microbiol. 2017;25(12):1016-26.

23. Zubair M, Malik A, Ahmad J. Clinico-bacteriology and risk factors for the diabetic foot infection with multidrug resistant microorganisms in north India. Biol Med. 2011;2(4): 22-34.

24. Ghada E, Ghada M. Characterization of Carbapenem Resistant Acinetobacter baumannii causing Ventilator associated Pneumonia in ICUs of Zagazig University Hospitals, Egypt. Inte J Curr Microbiol App Sci. 2016;5(12):660-71.

25. Mirani ZA, Aziz M, Khan MN, et al. Biofilm formation and dispersal of Staphylococcus aureus under the influence of oxacillin. Microb Pathog. 2013;61:66-72.

26. Marjorie de Oliveira A, Meg da Silva F, Filho B, et al. Inhibition and removal of staphylococcal biofilms using Moringa oleifera Lam. aqueous and saline extracts. J Environ Chem Eng. 2018;6(2):2011-6.

27. Oh E, Bae J, Kumar A, et al. Antioxidant-based synergistic eradication of methicillin-resistant Staphylococcus aureus (MRSA) biofilms with bacitracin. Int J Antimicrob Agents. 2018; 52(1):96-9. 